\title{
Decentralized Event-triggered Medium Access Control for Networked Control Systems
}

\author{
Mohammad H. Mamduhi, Maximilian Kneissl and Sandra Hirche ${ }^{1}$
}

\begin{abstract}
In practice often multiple control applications share a communication channel, requiring a smart and scalable scheduling mechanism to coordinate the access to the capacitylimited communication medium. In this paper, we propose a decentralized event-triggered medium access control (MAC) for multiple feedback control loops which are coupled through a capacity-limited communication medium. The individual control loops are assumed to be linear time-invariant (LTI) with stochastic heterogeneous plants. Noisy state measurements from local sensors are transmitted through a shared communication medium to their respective control units. Due to capacity limitations in the shared communication channel, not all sensors are allowed to transmit simultaneously. To allocate the scarce resources, a decentralized MAC which prioritizes the channel access according to a real-time error-dependent measure, is introduced. The prioritization is orchestrated via a combined deterministic and probabilistic mechanism aiming at the efficient allocation of the limited capacity. We study stability of the described multi-loop NCS under the proposed MAC design in terms of Lyapunov stability in probability (LSP). It is demonstrated that the collision rate remains low by properly tuning the MAC parameters. Numerical results show that the proposed MAC design significantly outperforms conventional time-triggered and random access schemes, while its performance closely follows the centralized TOD approach.
\end{abstract}

\section{INTRODUCTION}

Networked control systems such as industrial automation, smart energy grids, and autonomous vehicular systems are characterized by multitude of feedback control loops that are closed over a shared communication infrastructure. This poses novel challenges for the communication and control system design to support such NCSs with stringent realtime requirements [1]. The introduction of shared data transmission media imposes several design challenges such as capacity limitation, congestion, data loss, collisions, and latency [2]. These phenomena have negative impacts on control performance and may even destabilize the overall NCS. Traditionally, control systems employ time-triggered schemes with periodic sampling. There has been an increased interest recently in networked control approaches which take into account resource limitations leading to novel scheduling schemes for efficient data transmission. Since the seminal work [3] numerous results have shown that event-triggered control and scheduling approaches outperform time-triggered schemes as they often achieve the same control performance while consuming significantly less of the resource [4]-[8].

\footnotetext{
${ }^{1}$ M. H. Mamduhi, M. Kneissl, and S. Hirche are with the Chair of Information-Oriented Control, Technical University of Munich, Arcisstraße 21, D-80290 München, Germany http://www.itr.ei.tum.de, \{mh.mamduhi, max.kneissl, hirche\}etum.de
}

Time-triggered schemes sample the data at a fixed temporal schedule; their reconfiguration is computationally expensive, i.e. they are not scalable. Event-based strategies sample the data whenever a pre-specified event is triggered. Typically, the event depends on the system state and can be further adjusted based on scarcity of resource and channel situation [8].

The majority of the results study event-triggered sampling for single-loop networked control systems, while multi-loop NCSs over shared communication resources have attained little attention with some exceptions in [9]-[11]. In addition, it is mainly assumed that perfect state information is available for the event-trigger. However, in practice, often only noisy measurements are accessible. The event-trigger can be obtained by formulating the design as an optimal stochastic control problem [7], [10], [12]. These works show that eventtriggered sampling outperforms time-triggered schemes also in the multiple-loop NCSs. However, the communication models in these works are idealized, i.e. there is no data packet collision and no transmission delay. Prioritization in MAC strategies is introduced in [13] with the try-oncediscard (TOD) protocol. Based on current measurement data, TOD prioritizes transmissions by choosing the measurement with the largest discrepancy between its actual and estimated values, while the other measurements are discarded. TOD is a centralized scheme, i.e. not scalable. Stochastic prioritybased protocols [14]-[17] are shown to improve scalability and may also cope with communication unreliability. However, the designs are either centralized [14], or rely on having access to perfect state information, i.e. they assume noiseless sensors measure state values perfectly at every time-step [8].

In this paper, we introduce a decentralized event-triggered MAC scheme for multi-loop NCSs consisting of stochastic LTI plants sharing a limited-capacity communication channel. The proposed MAC scheme allocates the limited capacity based on the real-time conditions of the feedback loops leading to an improved overall NCS performance. The MAC protocol has a deterministic feature in form of a threshold policy, and a probabilistic feature, via a state-dependent biased randomization. The considered eventtrigger is considered as the discrepancy between the noisy measurements and the estimated state. If a local event is triggered, the current measurements are sent for transmission, otherwise, that sub-system does not attempt to transmit. The sub-systems attempting transmissions compete for the limited resource in probabilistic fashion such that the larger their local error is, the higher the chance of transmission becomes. We study stability properties of such NCSs under the proposed MAC scheme and show the overall NCS 


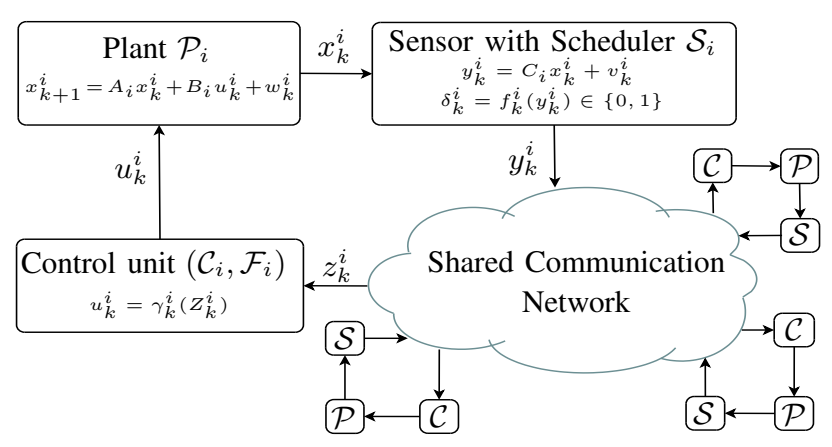

Fig. 1. Schematic of a shared resource multi-loop NCS.

stability in terms of LSP.

In the remainder of this paper, Section II presents the problem formulation and introduces the MAC strategy. Stability of the described NCS is discussed in Section III. Simulation results and discussions are presented in Section IV.

Notation: The Euclidean norm, conditional expectation, and conditional probability are denoted by $\|\cdot\|_{2}, \mathrm{E}[\cdot \cdot \cdot]$, and $\mathrm{P}[\cdot \mid \cdot \cdot]$, respectively. The trace operator is represented by $\operatorname{tr}(\cdot)$. A random vector $X$ from a multivariate Gaussian distribution with mean vector $\mu$ and covariance matrix $\Sigma$ is denoted by $X \sim \mathcal{N}(\mu, \Sigma)$. Superscripts and subscripts for state vectors indicate the belonging sub-system, and time instance, respectively. For matrices, subscripts indicate the belonging systems, while superscripts represent the matrix power.

\section{PROBLEM STATEMENT}

The NCS considered in this paper consists of $N$ heterogeneous physically independent control loops coupled through a shared communication channel subject to capacity limitations. Each control loop $i \in\{1, \ldots, N\}$ is composed of an LTI stochastic process $\mathcal{P}_{i}$, sensors $\mathcal{S}_{i}$, and a control unit which itself includes a Kalman filter $\mathcal{F}_{i}$ and a state feedback controller $\mathcal{C}_{i}$, see Fig. 1. The controlled sub-system $i$ can be described by the following discrete-time stochastic equations

$$
\begin{aligned}
x_{k+1}^{i} & =A_{i} x_{k}^{i}+B_{i} u_{k}^{i}+w_{k}^{i}, \\
y_{k}^{i} & =C_{i} x_{k}^{i}+v_{k}^{i},
\end{aligned}
$$

where $x_{k}^{i} \in \mathbb{R}^{n_{i}}, u_{k}^{i} \in \mathbb{R}^{m_{i}}, A_{i} \in \mathbb{R}^{n_{i} \times n_{i}}$ and $B_{i} \in \mathbb{R}^{n_{i} \times m_{i}}$ describe the $i^{\text {th }}$ sub-system state vector, control input, system matrix, and input matrix, respectively. For the ease of derivations, we assume $C_{i} \in \mathbb{R}^{n_{i} \times n_{i}}$ and $C_{i}^{-1}$ exists. The pairs $\left(A_{i}, B_{i}\right)$ and $\left(A_{i}, C_{i}\right)$ are assumed to be controllable, and observable, respectively. System noise and sensor noise are i.i.d. sequences with sample realizations $w_{k}^{i} \sim \mathcal{N}\left(0, W_{i}\right)$ and $v_{k}^{i} \sim \mathcal{N}\left(0, V_{i}\right)$, respectively. The initial state $x_{0}^{i}$ is randomly selected from an arbitrary finite-variance distribution.

Due to channel capacity limitation, not all sensor stations can transmit at the same time, thus, scheduling units are integrated in each station to determine whether a transmission is feasible. The scheduling decision at each time-step $k$ is taken locally, and is denoted by the binary variable $\delta_{k}^{i} \in\{0,1\}$ as:

$$
\delta_{k}^{i}= \begin{cases}1, & y_{k}^{i} \text { sent through the channel } \\ 0, & y_{k}^{i} \text { blocked. }\end{cases}
$$

We assume a lossless communication channel, i.e. there is no externally caused packet loss. However, packets from different control loops may collide during the medium access. Acknowledgment signals are broadcasted via an errorfree reserved link so every station is informed if a collision takes place. This can be realized in e.g. TCP-like protocols. We assume that if a collision is detected, the involved subsystems are discarded with no chance of re-transmission. The variable $\gamma_{k}^{i}$ is the collision indicator at a time-step $k$ as

$$
\gamma_{k}^{i}= \begin{cases}1, & y_{k}^{i} \text { successfully received } \\ 0, & y_{k}^{i} \text { collided. }\end{cases}
$$

System state values are estimated at the control units by the local Kalman filters, and the control inputs are then computed by the local feedback controllers. We define $\mathcal{I}_{k}^{i}=$ $\left\{\theta_{0}^{i}, y_{0}^{i}, \ldots, \theta_{k}^{i}, y_{k}^{i}\right\}$ as the history of received signals at the control side $\mathcal{C}_{i}$, where $\theta_{k}^{i}=\delta_{k}^{i} \gamma_{k}^{i}$. Then, the control input $u_{k}^{i}$ is computed by the following state-feedback law described by a measurable and causal mapping of past observations as

$$
u_{k}^{i}=-L_{i} \mathrm{E}\left[x_{k}^{i} \mid \mathcal{I}_{k}^{i}\right]=-L_{i} \hat{x}_{k}^{i},
$$

where, $L_{i} \in \mathbb{R}^{m_{i} \times n_{i}}$ is a stabilizing feedback gain. Depending on whether new sensory measurements are arriving, we compute the state estimate $\hat{x}_{k}^{i}$ via the Kalman filter, as

$$
\hat{x}_{k}^{i}=\hat{x}_{k}^{i-}+\theta_{k}^{i} K_{k}^{i}\left(y_{k}^{i}-C_{i} \hat{x}_{k}^{i-}\right)
$$

with the a priori state estimate $\hat{x}_{k}^{i-}$, and estimate error covariance $P_{k}^{i-}=\mathrm{E}\left[\left(x_{k}^{i}-\hat{x}_{k}^{i-}\right)\left(x_{k}^{i}-\hat{x}_{k}^{i-}\right)^{\top}\right]$ given as follows

$$
\begin{aligned}
& \hat{x}_{k+1}^{i-}=A_{i} \hat{x}_{k}^{i}+B_{i} u_{k}^{i}, \\
& P_{k+1}^{i-}=A_{i} P_{k}^{i} A_{i}^{\top}+W_{i},
\end{aligned}
$$

and the optimal Kalman gain $K_{k}^{i}$ and the a posteriori estimate error covariance $P_{k}^{i}=\mathrm{E}\left[\left(x_{k}^{i}-\hat{x}_{k}^{i}\right)\left(x_{k}^{i}-\hat{x}_{k}^{i}\right)^{\top}\right]$ as

$$
\begin{aligned}
K_{k}^{i} & =P_{k}^{i-} C_{i}^{\top}\left(C_{i} P_{k}^{i-} C_{i}^{\top}+V_{i}\right)^{-1}, \\
P_{k}^{i} & =P_{k}^{i-}-K_{k}^{i}\left(C_{i} P_{k}^{i-} C_{i}^{\top}+V_{i}\right) K_{k}^{i^{\top}},
\end{aligned}
$$

with the initial conditions $\hat{x}_{0}^{i}=0$ and $P_{0}^{i}=0$. It follows from (1)-(3) that $\delta_{k}^{i}=0$ leads to a model-based estimate of $x_{k}^{i}$, i.e. $\hat{x}_{k}^{i}=\hat{x}_{k}^{i-}=\left(A_{i}-B_{i} L_{i}\right) \hat{x}_{k-1}^{i}$. The estimate is well-behaved as the gain $L_{i}$ is stabilizing, and $\left(A_{i}, B_{i}\right)$ is stabilizable.

We introduce the network-induced error $e_{k}^{i}$ for each subsystem $i \in\{1, \ldots, N\}$, at every time-step $k$ as the difference between measurements and state estimates at the controller

$$
e_{k}^{i}:=y_{k}^{i}-\hat{y}_{k}^{i},
$$

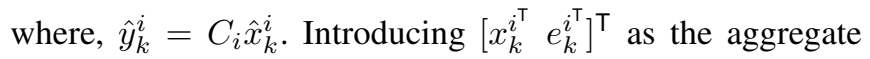
state of sub-system $i$, it is straightforward to derive the following state dynamics according to (1)-(6)

$$
\begin{aligned}
x_{k+1}^{i} & =\left(A_{i}-B_{i} L_{i}\right) x_{k}^{i}+B_{i} L_{i} C_{i}^{-1}\left(e_{k}^{i}-v_{k}^{i}\right)+w_{k}^{i}, \\
e_{k+1}^{i} & =\left(I_{n_{i}}-\theta_{k+1}^{i} C_{i} K_{k+1}^{i}\right)\left[C_{i} A_{i} C_{i}^{-1}\left(e_{k}^{i}-v_{k}^{i}\right)\right] \\
& +\left(I_{n_{i}}-\theta_{k+1}^{i} C_{i} K_{k+1}^{i}\right)\left[C_{i} w_{k}^{i}+v_{k+1}^{i}\right],
\end{aligned}
$$

It follows from (8) that evolution of the error state $e_{k}^{i}$ is independent of the system state $x_{k}^{i}$. This enables us to take an emulation-based control approach and choose a stabilizing controller a priori. The control inputs are computed 
according to this law at triggering times with updated state information, and in between based on the model-based state estimation. Stabilizability of pairs $\left(A_{i}, B_{i}\right)$ ensures that the closed-loop matrix $\left(A_{i}-B_{i} L_{i}\right)$ is stable. It follows from (7) that the aggregate state $\left[x_{k}^{i^{\top}} e_{k}^{i^{\dagger}}\right]^{\top}$ is stable if $e_{k}^{i}$ is stable.

Remark 1: In this paper we assume that output matrices are invertible. Relaxing this assumption urges the inclusion of additional observers which results in an additional observation error. Since the pairs $\left(A_{i}, C_{i}\right)$ are observable for all $i$ 's, stabilizing observer gains exist such that stability of the closed-loop system, including the observer state, is ensured in the absence of the capacity constraint. It is straightforward to show that, the evolution of network-induced error defined in (6) remains independent from the observer state and hence the scheduling process remains unaffected. The relaxation to arbitrary output matrices $C_{i}$, i.e. true output feedback control, is not considered here due to ease of derivations and space limitations. The essential techniques to show stability, however, extend to this case as well.

\section{A. Decentralized MAC Architecture}

Assume that the sampling interval of the communication network (micro slot), is much finer than the one of the control systems (macro slot). Further, assume $T$ is the sampling period of the control systems, then between two consecutive macro time slots $k T \rightarrow(k+1) T$, the micro slots are distributed as $\{k T, k T+\tau, \ldots, k T+(h-1) \tau, k T+h \tau\}$, where $\tau$ is the temporal duration of each micro slot and $h \gg 1$ denotes the number of micro slots within one macro slot, hence $T=h \tau$. We ideally assume that upon granting the channel access to a sensor station, noisy measurements are transmitted within the same control period. We additionally assume the channel is error-free, and sampling delays are negligible.

Having a decentralized architecture, the $i^{\text {th }}$ control and scheduling units are provided with only local information $A_{i}, B_{i}, C_{i}, W_{i}, V_{i}, L_{i}, K_{k}^{i}, \lambda_{i}, Q_{i}, \mathcal{I}_{k}^{i}$ and the distribution of $x_{0}^{i}$, where $\lambda_{i}$ and $Q_{i}$ denote pre-assigned error threshold, and positive definite weight matrix, respectively. The vector $y_{k^{\prime}+1}^{i}$ becomes eligible to enter the competition for the channel with the other control systems at a time-step $k^{\prime}+1$ only if the square weighted norm of $e_{k^{\prime}}^{i}$ exceeds the predesigned local threshold $\lambda_{i}$, otherwise it is discarded. Hence

$$
\mathrm{P}\left[\delta_{k^{\prime}+1}^{i}=1 \mid e_{k^{\prime}}^{i}\right]=0 \quad \text { if } \quad\left\|e_{k^{\prime}}^{i}\right\|_{Q_{i}}^{2} \leq \lambda_{i}
$$

where, $\left\|e_{k^{\prime}}^{i}\right\|_{Q_{i}}^{2}:=e_{k^{\prime}}^{i^{\top}} Q_{i} e_{k^{\prime}}^{i}$. We introduce $\mathcal{G}_{k^{\prime}+1}$ as the set of sensor stations cleared to transmit at time-step $k^{\prime}+1$, i.e. for each $i \in\{1, \ldots, N\}, i \in \mathcal{G}_{k^{\prime}+1}$, if $\left\|e_{k^{\prime}}^{i}\right\|_{Q_{i}}^{2}>\lambda_{i}$.

For the ease of notation, we assume that only one sensor station can transmit within every macro time-step, i.e.

$$
\sum_{i=1}^{N} \delta_{k^{\prime}}^{i}=1
$$

The following results can be readily generalized for $c$ dedicated channels, i.e. $\sum_{i=1}^{N} \delta_{k^{\prime}}^{i}=c<N$. A transmission can be commenced at any of the $h$ micro slots, within one macro slot, i.e. $\{0, \tau, 2 \tau, \ldots,(h-1) \tau\}$, only if the channel is sensed idle. We define a random variable $\nu_{k^{\prime}}^{i}$, called

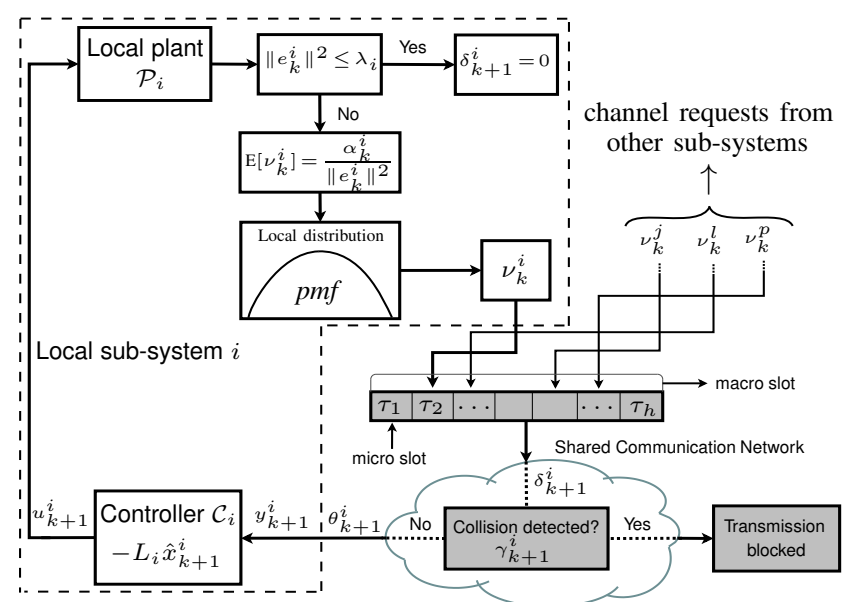

Fig. 2. NCS architecture with decentralized MAC algorithm.

waiting time, to determine the commence time. Thereby $\nu_{k^{\prime}}^{i} \in\{0, \tau, 2 \tau, \ldots,(h-1) \tau\}$ holds. Waiting times are chosen independently for each sub-system and at each time-step and denote the time duration a sensor station $i \in \mathcal{G}_{k^{\prime}+1}$ waits and then starts sensing the channel, i.e. at $k^{\prime} T+\nu_{k^{\prime}}^{i}$. Such a scenario can be implemented as a one-time back-off algorithm within, e.g. CSMA-based protocol. We propose a mechanism to select the local waiting times in a probabilistically prioritized fashion based on the real-time conditions of the local error states. First, we define independent local discrete distributions with finite-variance concave probability mass functions ( $p m f$ ), where the outcomes are the micro slots between $k^{\prime} \rightarrow k^{\prime}+1$. The waiting time $\nu_{k^{\prime}}^{i}$ is then randomly selected from the given local pmf with the following errordependent expected value

$$
\mathrm{E}\left[\nu_{k^{\prime}}^{i}\right]=\frac{\alpha_{k^{\prime}}^{i}}{\left\|e_{k^{\prime}}^{i}\right\|_{Q_{i}}^{2}},
$$

where, $\alpha_{k^{\prime}}^{i}$ is a local design parameter to tune the shape of the pmf, which consequently affects the collision rate. The concavity of the local $p m f s$ emphasizes the prioritized character of the MAC protocol as it ensures that the waiting times are more likely selected around the mean (11). Thus, the higher the local error of a sub-system is, the shorter the selected waiting time is expected to be. Finally, the sensor station associated with the shortest waiting time transmits, and the other stations discard. The same process repeats at the start of each macro slot incorporating the updated error norms. Hence, considering (10), the probability that sensor station $i \in \mathcal{G}_{k^{\prime}+1}$ transmits at time-step $k^{\prime}+1$ is as follows:

$$
\mathrm{P}\left[\delta_{k^{\prime}+1}^{i}=1 \mid e_{k^{\prime}}^{i}\right]=\mathrm{P}\left[\nu_{k^{\prime}}^{i}<\nu_{k^{\prime}}^{l}\right], \quad \forall l \in \mathcal{G}_{k^{\prime}+1}, l \neq i .
$$

According to (12), a collision occurs if at least two schedulers select identical waiting times. Selecting the waiting times in the introduced random fashion reduces the collision rate since it decreases the chance of collision between sub-systems with similar error values. On the other hand, assuming many sub-systems with high error values leads to concentration of local pmfs across early micro slots, which increases the chance of collision. To avoid so, parameter $\alpha_{k^{\prime}}^{i}$ can be properly adjusted according to broadcasted channel 
feedback which contains information about latest traffic and collisions, to place the mean of the $p m f$ s farther than the congested area. We will discuss this in Section IV.

Remark 2: The error thresholds are incorporated to improve performance of the scheduling process by efficient allocation of limited resources. Intuitively, setting the thresholds very low allows the sub-systems with negligible error values to take part in the channel competition. Since channel access is granted randomly, those sub-systems may transmit instead of sub-systems with higher priorities. On the other hand, setting the thresholds high implies that sub-systems with relatively high errors are blocked, which also leads to performance deficiency. Hence, there should be optimal values of error thresholds which maintain a balance between optimizing performance and maximizing transmission chance for sub-systems with critical real-time conditions [18]. Deriving optimal thresholds is out of this paper's scope.

\section{STABILITY ANALYSIS}

Definition 1: (Lyapunov Stability in Probability, [19]) A discrete linear system with state vector $x_{k}$ possesses LSP if for given $\varepsilon_{i}, \varepsilon_{i}^{\prime}>0$, exists $\rho_{i}\left(\varepsilon_{i}, \varepsilon_{i}^{\prime}\right)>0$ s. t. $\left|x_{0}\right|<\rho_{i}$ implies

$$
\lim _{k \rightarrow \infty} \sup \mathrm{P}\left[x_{k}^{\top} x_{k} \geq \varepsilon_{i}^{\prime}\right] \leq \varepsilon_{i} .
$$

Recall that the emulation-based control approach ensures the closed-loop matrix $\left(A_{i}-B_{i} L_{i}\right)$ is stable. We show in the following lemma that the existence of stabilizing gains $L_{i}$ 's ensures a sub-system $i$ with the aggregate state $\left[x_{k}^{i^{\top}} e_{k}^{i^{\top}}\right]^{\top}$ is LSP, only if the error state $e_{k}^{i}$ is bounded in probability.

Lemma 1: For a control loop with state vector $\left[x_{k}^{i^{\top}} e_{k}^{i^{\top}}\right]^{\top}$, described in (7) and (8), respectively, the LSP condition (13) is equivalently satisfied if $\xi_{i}^{\prime}>0$ and $0 \leq \xi_{i} \leq \varepsilon_{i}$ exists s. t.

$$
\lim _{k \rightarrow \infty} \sup \mathrm{P}\left[e_{k}^{i^{\top}} e_{k}^{i} \geq \xi_{i}^{\prime}\right] \leq \xi_{i}
$$

Proof: The system state $x_{k}^{i}$ evolves according to (7), where the closed-loop matrix $\left(A_{i}-B_{i} L_{i}\right)$ is Hurwitz. In addition, the term $B_{i} L_{i} C_{i}^{-1}\left(e_{k}^{i}-v_{k}^{i}\right)$ in (7) represents the consequence of limited resources on the system dynamics, which cannot be affected by the control input $u_{k}^{i}$. It follows from Markov's inequality that for $\zeta_{i}^{\prime}>0$

$$
\begin{aligned}
& \mathrm{P}\left[\left(e_{k}^{i}-v_{k}^{i}\right)^{\top}\left(e_{k}^{i}-v_{k}^{i}\right) \geq \zeta_{i}^{\prime}\right] \leq \frac{\mathrm{E}\left[\left(e_{k}^{i}-v_{k}^{i}\right)^{\top}\left(e_{k}^{i}-v_{k}^{i}\right)\right]}{\zeta_{i}^{\prime}} \\
& =\frac{\mathrm{E}\left[\left\|e_{k}^{i}-v_{k}^{i}\right\|_{2}^{2}\right]}{\zeta_{i}^{\prime}} \leq \frac{\mathrm{E}\left[\left\|e_{k}^{i}\right\|_{2}^{2}\right]+\operatorname{tr}\left(V_{i}\right)+2 \sqrt{\operatorname{tr}\left(V_{i}\right) \mathrm{E}\left[\left\|e_{k}^{i}\right\|_{2}^{2}\right]}}{\zeta_{i}^{\prime}}
\end{aligned}
$$

where the last inequality follows from $\mathrm{E}\left[\left\|e_{k}^{i}-v_{k}^{i}\right\|_{2}^{2}\right]=$ $\mathrm{E}\left[\left\|e_{k}^{i}\right\|_{2}^{2}\right]+\mathrm{E}\left[\left\|v_{k}^{i}\right\|_{2}^{2}\right]-2 \operatorname{Cov}\left(e_{k}^{i^{\top}}, v_{k}^{i^{\top}}\right)$, and $\operatorname{Cov}\left(e_{k}^{i^{T^{\top}}}, v_{k}^{i^{\top}}\right) \leq$ $\sqrt{\operatorname{Var}\left(v_{k}^{i}\right) \operatorname{Var}\left(e_{k}^{i}\right)}$. The closed-loop system $\left(A_{i}-B_{i} L_{i}\right) x_{k}^{i}+$ $w_{k}^{i}$ is bounded in expectation and consequently possesses LSP, since $\left(A_{i}-B_{i} L_{i}\right)$ is Hurwitz and $\mathrm{E}\left[x_{0}^{i}\right]$ is bounded, while $w_{k}^{i} \sim \mathcal{N}\left(0, W_{i}\right)$. Thus, showing $\mathrm{E}\left[e_{k}^{i^{\top}} e_{k}^{i}\right]$ is bounded, ensures $\lim _{k \rightarrow \infty} \sup \mathrm{P}\left[\left(e_{k}^{i}-v_{k}^{i}\right)^{\top}\left(e_{k}^{i}-v_{k}^{i}\right) \geq \zeta_{i}^{\prime}\right] \leq \zeta_{i}$ with $\zeta_{i}=\frac{\mathrm{E}\left[\left\|e_{k}^{i}\right\|_{2}^{2}\right]+\operatorname{tr}\left(V_{i}\right)+2 \sqrt{\operatorname{tr}\left(V_{i}\right) \mathrm{E}\left[\left\|e_{k}^{i}\right\|_{2}^{2}\right]}}{\zeta_{i}^{\prime}}$. This implies boundedness of $\mathrm{E}\left[x_{k+1}^{i^{\top}} x_{k+1}^{i}{ }^{\zeta_{i}}\right]$ which assures $\varepsilon_{i} \geq \xi_{i}$ and $\varepsilon_{i}^{\prime}>0$ exist such that $\lim _{k \rightarrow \infty} \sup \mathrm{P}\left[x_{k+1}^{i^{\top}} x_{k+1}^{i} \geq \varepsilon_{i}^{\prime}\right] \leq \varepsilon_{i}$, and LSP of the system with state $\left[x_{k}^{i^{\dagger}} e_{k}^{i^{\top}}\right]^{\top}$ then readily follows.

Note that the random variables $e_{k}^{i}$ and $v_{k}^{i}$ are statistically dependent, according to (8), and thus the error state $e_{k}^{i}$ is not inherently Markovian. However, Markovianity of a process state is not necessary to show the LSP, see Definition 1.

As individual loops operate independently, we define the overall NCS state $\left[x_{k}^{\top} e_{k}^{\top}\right]^{\top}$ by stacking local states $\left[x_{k}^{i^{\top}} e_{k}^{i^{\top}}\right]^{\top}$ from all $i \in\{1, \ldots, N\}$. Therefore, ensuring LSP for all subsystems guarantees the overall NCS with the augmented state $\left[x_{k}^{\top} e_{k}^{\top}\right]^{\top}$ is LSP. In the other words, existence of $\xi, \xi^{\prime}>0$ such that $\lim _{k \rightarrow \infty} \sup \mathrm{P}\left[e_{k}^{\top} e_{k} \geq \xi^{\prime}\right] \leq \xi$, implies $\varepsilon, \varepsilon^{\prime}>0$ exist such that $\lim _{k \rightarrow \infty} \sup \mathrm{P}\left[x_{k+1}^{\top} x_{k+1} \geq \varepsilon^{\prime}\right] \leq \varepsilon$. Incorporating weight matrix $Q=\operatorname{diag}\left(Q_{i}\right)$, LSP condition (14) becomes

$$
\lim _{k \rightarrow \infty} \sup \mathrm{P}\left[e_{k}^{\top} Q e_{k} \geq \bar{\xi}^{\prime}\right] \leq \bar{\xi}
$$

According to Markov's inequality, we can instead focus on the expectation of weighted quadratic error norm, i.e.

$$
\mathrm{P}\left[e_{k}^{\top} Q e_{k} \geq \bar{\xi}^{\prime}\right] \leq \frac{\mathrm{E}\left[e_{k}^{\top} Q e_{k}\right]}{\bar{\xi}^{\prime}}=\frac{\sum_{i=1}^{N} \mathrm{E}\left[\left\|e_{k}^{i}\right\|_{Q_{i}}^{2}\right]}{\bar{\xi}^{\prime}}
$$

Due to the constraint (10), boundedness of (16) is not always guaranteed over one time-step horizon, i.e. from $k \rightarrow k+1$. The following example illustrates this observation.

Illustrative Example: Let an NCS consist of two identical scalar control loops, i.e. $A_{1}=A_{2}=\bar{a}$, competing for the sole transmission possibility at time-step $k+1$. For the purpose of illustration, let $Q_{1}=Q_{2}=1, \lambda_{1}=\lambda_{2}=0, \alpha_{k}^{1}=\alpha_{k}^{1}=\bar{\alpha}$, $C_{1}=C_{2}=1, V_{1}=V_{2}=0$ and $e_{k}^{1}=e_{k}^{2}=\bar{e}_{k}$ be the initial state. Consider that both sub-systems select their waiting times $\nu_{k+1}^{1}$ and $\nu_{k+1}^{2}$ from identical $p m f$ s, with the same mean $\frac{\bar{\alpha}^{2}}{\left\|\bar{e}_{k}\right\|_{2}^{2}}$, according to (11). Hence, each sub-system has $50 \%$ chance of channel access. It follows from (8) that

$$
\begin{aligned}
& \sum_{i=1,2} \mathrm{E}\left[\left\|e_{k+1}^{i}\right\|_{2}^{2} \mid e_{k}^{i}\right]=\sum_{i=1,2} \mathrm{E}\left[\left\|\left(1-\theta_{k+1}^{i}\right)\left(a \bar{e}_{k}+w_{k}^{i}\right)\right\|_{2}^{2} \mid \bar{e}_{k}\right] \\
& =\sum_{i=1,2} \frac{1}{2} \mathrm{E}\left[\left\|a \bar{e}_{k}+w_{k}^{i}\right\|_{2}^{2} \mid \bar{e}_{k}\right]=\frac{\operatorname{tr}\left(W_{1}+W_{2}\right)}{2}+a^{2}\left\|\bar{e}_{k}\right\|_{2}^{2},
\end{aligned}
$$

which is not bounded for arbitrary initial state $\bar{e}_{k}$ and system parameter $\bar{a}$. This is an inevitable effect of the capacity limitation. The absence of new measurements results in the expected growth of local errors. Generally, boundedness of the error state in expectation can only be obtained over an interval during which all sub-systems are expected to transmit. Due to the constraint (10), the interval of length $N$ provides enough transmission chances, i.e. $N$, for all sub-systems. It should however be noticed that having $N$ transmission chances does not at all guarantee that all $N$ sub-systems transmit. In what follows, we will show that (15) can be guaranteed over an operational interval of length $N$, i.e. starting from time-step $k$, we take the interval $[k, k+N]$. Before proceeding to the main theorem of this paper, we compute the probability that all transmission attempts from every sensor station fail due to successive collisions, over the interval $[k, k+N]$. Due to the decentralized nature of 
the MAC protocol (9) and (12), there exists a non-zero probability that collisions take place successively over the interval $[k, k+N]$, and all sub-systems remain open-loop. This occurs if at least two sub-systems select identical waiting times at every time-step over $[k, k+N]$. Assume a sub-system $i$ selects a waiting time $\nu_{k^{\prime}}^{i}=s_{k^{\prime}}^{i} \tau$ at time-step $k^{\prime}$, with $s_{k^{\prime}}^{i} \in\{1, \ldots, h-1\}$. We can compute the probability that another sub-system $j \neq i$ selects exactly the identical waiting time $\nu_{k^{\prime}}^{j}=s_{k^{\prime}}^{i} \tau$. We know from the probability theory that $\mathrm{E}\left[\nu_{k^{\prime}}^{j}\right]=\sum_{m=1}^{h-1} m \tau \mathrm{P}\left(\nu_{k^{\prime}}^{j}=m \tau\right)$. In addition, knowing that $\mathrm{E}\left[\nu_{k^{\prime}}^{j}\right]=\frac{\alpha_{k^{\prime}}^{j}}{\left\|e_{k^{\prime}}^{j}\right\|_{Q_{j}}^{2}}<\frac{\alpha_{k^{\prime}}^{j}}{\lambda_{j}}$, we can compute an upper-bound for the probability that the certain event $\nu_{k^{\prime}}^{j}=s_{k^{\prime}}^{i} \tau$ is selected:

$$
\begin{gathered}
\mathrm{P}\left(\nu_{k^{\prime}}^{j}=s_{k^{\prime}}^{i} \tau\right)=\frac{1}{s_{k^{\prime}}^{i} \tau}\left[\mathrm{E}\left[\nu_{k^{\prime}}^{j}\right]-\sum_{m=1, m \neq s_{k^{\prime}}^{i}}^{h-1} m \tau \mathrm{P}\left(\nu_{k^{\prime}}^{j}=m \tau\right)\right] \\
<\frac{1}{s_{k^{\prime}}^{i} \tau}\left[\frac{\alpha_{k^{\prime}}^{j}}{\lambda_{j}}-\sum_{m=1, m \neq s_{k^{\prime}}^{i}}^{h-1} m \tau \mathrm{P}\left(\nu_{k^{\prime}}^{j}=m \tau\right)\right] \leq \frac{\alpha_{k^{\prime}}^{j}}{s_{k^{\prime}}^{i} \tau \lambda_{j}} .
\end{gathered}
$$

Extending this for every pair of sub-systems $i$ and $j$, we find the upper-bound for probability of successive collisions as

$$
\mathrm{P}\left[\sum_{i=1}^{N} \theta_{k^{\prime}}^{i}=0, \forall k^{\prime}\right] \leq \prod_{k^{\prime}=k}^{k+N} \sum_{i=1}^{N} \sum_{j=1, j \neq i}^{N} \frac{\alpha_{k^{\prime}}^{j}}{s_{k^{\prime}}^{i} \tau \lambda_{j}}
$$

From (17), increasing the error thresholds lowers the successive collision rate, which is intuitive as it results in more sub-systems being out of channel access competition. Moreover, increasing $h$ reduces the collision probability as it increases the number of events $s_{k^{\prime}}^{i} \tau$, hence reducing the probability that a specific event is selected more than once. In addition, (17) provides an upper-bound for the probability that Lyapunov stability cannot be achieved, which motivates the selection of the stability notion, i.e. LSP, in this paper.

Theorem 1: Let a multi-loop NCS consist of $N$ heterogeneous LTI stochastic sub-systems with states and measurements given by (1), sharing a communication channel subject to the constraint (10). Under the control, estimation laws (2) and (3), and scheduling rule (9) and (12), the overall NCS with augmented state $\left[x_{k}^{\top}, e_{k}^{\top}\right]^{\top}$ possesses LSP for any positive $\lambda_{i}$ 's and $\alpha_{k}^{i}$ 's, and any positive definite $Q_{i}$ 's.

Proof: See Appendix.

\section{NUMERICAL RESULTS}

To evaluate the performance of the proposed approach, we consider a model of NCS with two different classes of scalar sub-systems, i.e. $\left\{c l_{1}, c l_{2}\right\}$. The state-space representation for the first class including unstable plants is $\left(A_{c l_{1}}, B_{c l_{1}}, C_{c l_{1}}\right)=$ $(1.25,1,1)$, while for the second class containing stable plants $\left(A_{\mathrm{cl}_{2}}, B_{\mathrm{cl}_{2}}, C_{\mathrm{cl}_{2}}\right)=(0.75,1,1)$. Each sub-system is steered via a dead-beat control law $L_{i}=\frac{A_{i}}{B_{i}}$. For simplicity, we select $Q_{i}=I$ for all $i$, and set $x_{0}^{c l_{1}}=x_{0}^{c l_{2}}=0$. The process noise and measurement noise are realized as $w_{k}^{i} \sim \mathcal{N}(0,1)$ and $v_{k}^{i} \sim \mathcal{N}(0,0.1)$, respectively. We conduct a Monte Carlo simulation with $2 \times 10^{5}$ samples and assume a channel capacity of $c=1$. The simulations are performed for a varying number of sub-systems $N \in\{2,4,6,8,10,12\}$,
TABLE I

DECENTRALIZED AND UNIFORM BI-CHARACTER SCHEDULING

\begin{tabular}{lcccccc}
\hline Number of plants $(\mathrm{N})$ & 2 & 4 & 6 & 8 & 10 & 12 \\
\hline \multicolumn{7}{c}{ decentralized bi-character } \\
\hline Error threshold $(\lambda)$ & 0.01 & 0.10 & 0.40 & 0.70 & 0.88 & 0.98 \\
aggregate error variance & 1.08 & 1.50 & 1.96 & 2.50 & 3.17 & 4.20 \\
collision rate $(\%)$ & 0.78 & 0.89 & 1.12 & 1.35 & 1.58 & 1.88 \\
\hline \multicolumn{7}{c}{ uniform bi-character } \\
\hline Error threshold $(\lambda)$ & 0.10 & 2.10 & 3.80 & 6.00 & 11.20 & 14.00 \\
aggregate error variance & 0.90 & 1.91 & 2.79 & 3.79 & 5.10 & 7.61 \\
collision rate $(\%)$ & 0.19 & 0.19 & 0.24 & 0.30 & 0.27 & 0.35 \\
\hline
\end{tabular}

with $\frac{N}{2}$ sub-systems belong to each class. Moreover, in every simulated NCS setting the same error thresholds $\lambda$ are applied for all sub-systems. The number of micro slots is set to $h=150$ and the waiting times are chosen from Poisson distributions with error-dependent mean (11). To tune the scaling factor $\alpha_{k^{\prime}}^{i}$, we utilize the feedback signal broadcasted from the channel, according to the following empirical law

$$
\alpha_{k^{\prime}}^{i}=q_{t} n_{t, k^{\prime}-1}+q_{c} n_{c, k^{\prime}-1},
$$

where $n_{t, k-1}$ is the traffic, i.e. the number of systems trying to transmit, and $n_{c, k-1}$ is the latest time collision rate, and they are accompanied by the weights $q_{t}$ and $q_{c}$, respectively.

Table I shows the resulting aggregate error variance of our proposed MAC for different number of sub-systems $N$, with the error thresholds $\lambda$ and the scaling weights $q_{t}=60$ and $q_{c}=34$. Furthermore, it provides the collision rates, which are shown to be low due to proper parameterization of the Poisson distributions. It also shows the results of a uniform bi-character realization, where sub-systems are deterministically excluded from channel access if their error is below the threshold $\lambda$, otherwise waiting times are chosen based on a uniform distribution. As expected, this leads to less collisions since the waiting times are selected by identical probabilities across the entire macro slot, however, it increases error variances as channel access is not prioritized.

Fig. 3 provides comparisons with the other commonly known protocols. The centralized TOD approach is considered as the lower bound as it leads to the best performance. For the CSMA, constant access probabilities are assigned to each sub-system depending on the system matrices, i.e. $\frac{\left\|A_{i}\right\|_{2}^{2}}{\sum_{j=1}^{N}\left\|A_{j}\right\|_{2}^{2}}$, which is already an improvement to standard CSMA with access probability is $\frac{1}{N}$. This approach yields an acceptable performance only for $N=2$. The performance of the proposed MAC protocol follows that of the TOD closely, which proves that the performance is almost preserved with the proposed decentralization. Moreover, it outperforms the TDMA, where a constant time-based order of transmission is assumed wherein only unstable plants are part of the schedule. The uniform bi-character comparison shows a decrease in collision rates, but error variances can not compete with the decentralized law for high number of systems.

Fig. 4 shows the impact of distribution scaling on the collision rate and aggregate error variance, for a typical NCS with $N=8$. The red $p d f$, where the mean of the scaling 


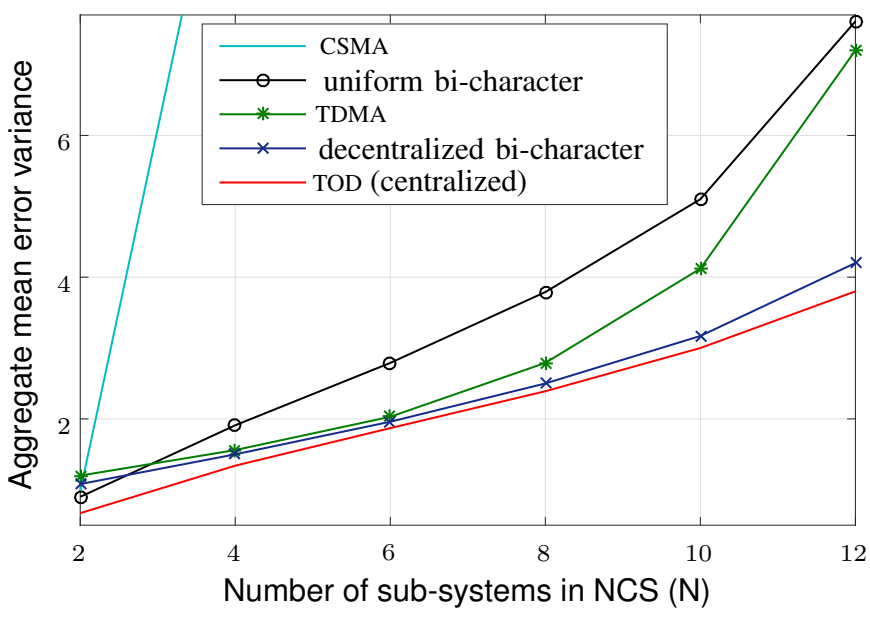

Fig. 3. Comparison of the aggregate error variances for a varying number of sub-systems and different MAC protocol realizations.

factor $\alpha_{k^{\prime}}^{i}$ equals 77.51, shows that the selected waiting times are concentrated at the beginning of the macro slot, which leads to a collision rate of $3.47 \%$, and error variance of 2.71. By tuning the parameters in (18) properly, the mean of $\alpha_{k^{\prime}}^{i}$ changes to 163 , which results in a better use of the entire channel range in one slot. This leads to less collisions $(1.35 \%)$ and consequently lower error variance $(2.50)$.

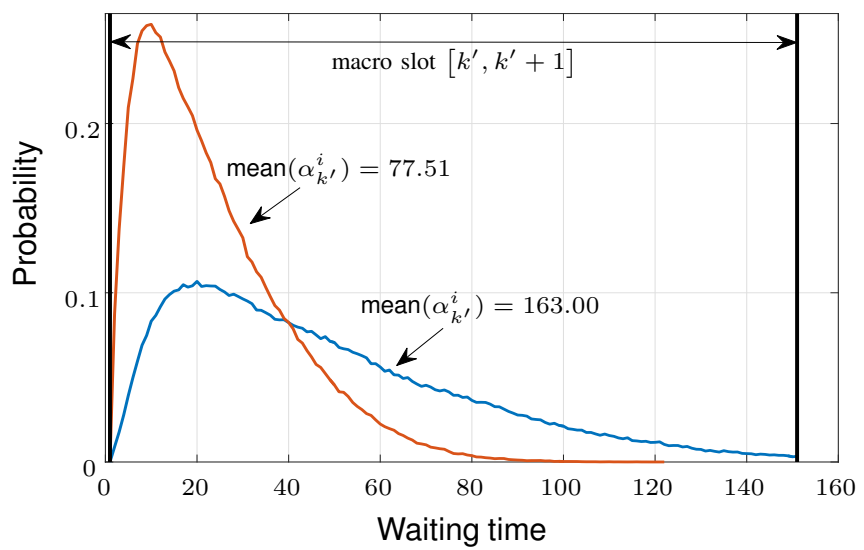

Fig. 4. $p d f$ of resulting waiting times with mean value $\alpha_{k^{\prime}}^{i}=77.51$ and $\alpha_{k^{\prime}}^{i}=163.00$, for NCS with $N=8$ sub-systems during one macro slot.

\section{CONCLUSION}

We propose a decentralized MAC strategy for multipleloop NCSs with shared communication resources. The decision that either a sensor station can transmit is performed by local schedulers, capable of real-time prioritization in both deterministic and probabilistic fashions. It is shown that the MAC strategy preserves stability of the overall NCS in terms of LSP, and in addition can be appropriately adjusted to cope with channel traffic. Simulation results illustrate the performance enhancement by the proposed error-dependent approach compared to the TDMA and CSMA protocols and non-error-dependent approach. Moreover, our approach performs closely to the centralized TOD approach.

\section{APPENDIX}

Proof: (Theorem 1) As discussed in Lemma 1, LSP of the NCS with augmented state $\left[x_{k}^{\top}, e_{k}^{\top}\right]^{\top}$ follows if $e_{k}$ satisfies
(14). Furthermore, the condition (14) can be satisfied by alternatively upper-bounding the expectation of the weighted error norm, according to (16). In addition, we discussed that the condition (14) is investigated over an interval with length $N$, i.e. $[k, k+N]$. First, we express the dynamic behavior of a local error state $e_{k}^{i}$ while transiting up to time-step $k+N$, starting at the reference time $k+r_{i}$, where $r_{i} \in[0, N-1]$ :

$$
\begin{aligned}
& e_{k+N}^{i}=\prod_{d_{1}=0}^{N-r_{i}-1}\left(\Delta_{k+N-d_{1}}^{i} \bar{A}_{i}^{c}\right)\left(e_{k+r_{i}}^{i}-v_{k+r_{i}}^{i}\right) \\
& \quad+\sum_{q_{1}=r_{i}}^{N-1} \prod_{d_{2}=0}^{N-q_{1}-2}\left(\Delta_{k+N-d_{2}}^{i} \bar{A}_{i}^{c}\right) \Delta_{k+q_{1}+1}^{i} C_{i} w_{k+q_{1}}^{i} \\
& \quad+\sum_{q_{2}=r_{i}+1}^{N-1} \prod_{d_{3}=0}^{N-q_{2}-1}\left(\Delta_{k+N-d_{3}}^{i} \bar{A}_{i}^{c}\right)\left(\Delta_{k+q_{2}}^{i}-I_{n_{i}}\right) v_{k+q_{2}}^{i} \\
& \quad+\Delta_{k+N}^{i} v_{k+N}^{i},
\end{aligned}
$$

where, $\Delta_{k^{\prime}}^{i}=I_{n_{i}}-\theta_{k^{\prime}}^{i} C_{i} K_{k^{\prime}}^{i}$, and $\bar{A}_{i}^{c}=C_{i} A_{i} C_{i}^{-1}$, and we define $\prod_{0}^{-1}(\cdot):=1$, and $\sum_{N}^{N-1}(\cdot):=0$. We first study the case that successive collisions occur over the interval $[k, k+N]$, i.e. $\theta_{k^{\prime}}^{i}=0$ for all $i \in\{1, \ldots, N\}$ at all timesteps $k^{\prime} \in[k, k+N]$. Let $r_{i}=0$, then it holds from (19) that $e_{k+N}^{i}=\bar{A}_{i}^{c^{N-1}}\left(e_{k}^{i}-v_{k}^{i}\right)+\sum_{q_{1}=0}^{N-1} \bar{A}_{i}^{c^{N-q_{1}-2}} C_{i} w_{k+q_{1}}^{i}+$ $v_{k+N}^{i}$. The probability that such a scenario occurs is computed in (17). Setting $\bar{\xi}^{\prime}=\sum_{i=1}^{N} \| \bar{A}_{i}^{c^{N-1}}\left(e_{k}^{i}-v_{k}^{i}\right)+$ $\sum_{q_{1}=0}^{N-1} \bar{A}_{i}^{c^{N-q_{1}-2}} C_{i} w_{k+q_{1}}^{i}+v_{k+N}^{i} \|_{Q_{i}}^{2}$, it then follows from (15) that $\bar{\xi}$ equals the right side of the inequality (17), i.e.,

$$
\sup _{e_{k}} \mathrm{P}\left[\sum_{i=1}^{N}\left\|e_{k+N}^{i}\right\|_{Q_{i}}^{2} \geq \bar{\xi}^{\prime}\right]<\prod_{k^{\prime}=k}^{k+N} \sum_{i=1}^{N} \sum_{j=1, j \neq i}^{N} \frac{\alpha_{k^{\prime}}^{j}}{s_{k^{\prime}}^{i} \tau \lambda_{j}} .
$$

In the rest of this proof, we assume that not all data packets collide over the interval $[k, k+N]$. The behavior of a subsystem $i$ while transiting from $k$ to $k+N$ can be characterized by one of the following three complementary and mutually exclusive occurrences, covering the entire sample space. First, a sub-system $i$ is in the set $\overline{\mathcal{G}}_{k^{\prime}+1}:=\mathbf{N}-\mathcal{G}_{k^{\prime}+1}$, at least in one occasion over $[k, k+N]$, where $\mathbf{N}$ is the set of all sub-systems $\{1, \ldots, N\}$. Second, a sub-system $i$ is always in $\mathcal{G}_{k^{\prime}+1}$, but has successfully transmitted at least once over $[k, k+N]$. Third, over the same period, a sub-system $i$ is always in $\mathcal{G}_{k^{\prime}+1}$, while it has never successfully transmitted. For the first scenario, denoted as $s_{1}$, assume that the timestep $k+r_{i}$ is the latest occasion where $i \in \overline{\mathcal{G}}_{k+r_{i}+1}$. This implies $\left\|e_{k+r_{i}}^{i}\right\|_{Q_{i}}^{2} \leq \lambda_{i}$. From (19), and due to the mutual statistical independence of $e_{k+r_{i}}^{i}, w_{k+q_{1}}^{i}, v_{k+q_{2}}^{i}$, and $v_{k+N}^{i}$, and in addition, knowing from (4) that $I-C_{i} K_{k^{\prime}}^{i}$ is positive definite, the following upper-bound is obtained for all $i \in s_{1}$

$$
\begin{aligned}
& \sum_{i \in s_{1}} \mathrm{E}\left[\left\|e_{k+N}^{i}\right\|_{Q_{i}}^{2} \mid e_{k}^{i}\right] \leq \\
& \sum_{i \in s_{1}}\left\|\bar{A}_{i}^{c^{N-r_{i}-1}}\right\|_{2}^{2} \mathrm{E}\left[\left\|e_{k+r_{i}}^{i}-v_{k+r_{i}}^{i}\right\|_{Q_{i}}^{2} \mid e_{k}^{i}\right]+ \\
& \sum_{i \in s_{1}} \sum_{q_{1}=r_{i}}^{N-1} \operatorname{tr}\left(Q_{i} W_{i}\right)\left\|\bar{A}_{i}^{c^{N-q_{1}-2}} C_{i}\right\|_{2}^{2}+\operatorname{tr}\left(Q_{i} V_{i}\right) \leq \\
& \sum_{i \in s_{1}}\left\|\bar{A}_{i}^{c^{N-r_{i}-1}}\right\|_{2}^{2}\left[\lambda_{i}+\operatorname{tr}\left(Q_{i} V_{i}\right)+2 \sqrt{\operatorname{tr}\left(Q_{i} V_{i}\right) \lambda_{i}}\right]+\zeta_{b}^{i}
\end{aligned}
$$

where, $\zeta_{b}^{i}$ stands for the third line of the above expression. LSP condition (15) is fulfilled according to (16) with $\bar{\xi}^{\prime}$ selected larger than (20), and $\bar{\xi}=\frac{\sum_{i \in s_{1}} \mathrm{E}\left[\left\|e_{k+N}^{i}\right\|_{Q_{i}}^{2} \mid e_{k}^{i}\right]}{\xi^{\prime}}<1$. 
For sub-systems $i$ within the second scenario $s_{2}$, assume $k+r_{i}$ is the latest time-step that $\theta_{k+r_{i}}^{i}=1$. Hence, $\theta_{k+r_{i i}}^{i}=0$ for all time-steps $r_{i i}$, with $r_{i}<r_{i i} \leq N$. It follows from (19)

$$
\begin{aligned}
& \sum_{i \in s_{2}} \mathrm{E}\left[\left\|e_{k+N}^{i}\right\|_{Q_{i}}^{2} \mid e_{k}^{i}\right] \leq \\
& \sum_{i \in s_{2}}\left\|\bar{A}_{i}^{c^{N-r_{i}-1}}\right\|_{2}^{2} \operatorname{tr}\left(Q_{i} C_{i} P_{k+r_{i}}^{i} C_{i}^{\mathrm{\top}}\right)+\zeta_{b}^{i}
\end{aligned}
$$

where, $P_{k+r_{i}}^{i}$ is the posteriori covariance matrix of estimation error $x_{k+r_{i}}^{i}-\hat{x}_{k+r_{i}}^{i}$. Upper-bound (21) follows from the Kalman filter properties ensuring that if $\theta_{k+r_{i}}^{i}=1$, then, $\mathrm{E}\left[e_{k+r_{i}}^{i}-v_{k+r_{i}}^{i}\right]=C_{i} \mathrm{E}\left[x_{k+r_{i}}^{i}-\hat{x}_{k+r_{i}}^{i}\right]=0$, and $\mathrm{E}\left[\left\|e_{k+r_{i}}^{i}-v_{k+r_{i}}^{i}\right\|_{Q_{i}}^{2}\right]=\operatorname{tr}\left(Q_{i} \operatorname{Cov}\left[e_{k+r_{i}}^{i}-v_{\underline{k}+r_{i}}^{i}\right]\right)$. Similarly, LSP condition (15) satisfies considering $\bar{\xi}^{\prime}$ selected to be larger than (21), and $\bar{\xi}=\frac{\sum_{i \in s_{2}} \mathrm{E}\left[\left\|e_{k+N}^{i}\right\|_{Q_{i}}^{2} \mid e_{k}^{i}\right]}{\xi^{\prime}}<1$.

For sub-systems $i \in s_{3}$, a uniform bound for $\mathrm{E}\left[\left\|e_{k+N}^{i}\right\|_{Q_{i}}^{2}\right]$ cannot be found since $\left\|e_{k^{\prime}}^{i}\right\|_{Q_{i}}^{2}>\lambda_{i}$ for all $k^{\prime} \in[k, k+N]$. However, as the introduced scenarios are mutually exclusive, one can compute the probability that a sub-system $i$ belongs to $s_{3}$. Intuitively, $i$ belongs to $s_{3}$ due to either successive channel busy, or having collisions whenever the channel is idle. Assume that whenever a collision is detected, a virtual control loop has successfully transmitted. This means at the time a collision occurs, $N$ real and one virtual sub-systems share the channel and the virtual sub-system transmits. The virtual loops are assumed to have the same dynamics as in (1). Assume that the channel experiences $m<N$ collisions over the interval $[k, k+N]$. Having $N+m$ sub-systems requires our operational interval to be extended to $\left[k, k_{m}\right]$, where $k_{m}=k+N+m$. For one sub-system $i$ to be in $s_{3}$, another sub-system, say $j$, should have re-transmitted at the final time $k_{m}$. Let $k+r_{j}<k_{m}$ denote the latest time at which $\theta_{k+r_{j}}^{j}=1$. The probability that $j$ re-transmits at $k_{m}$, in the presence of sub-system $i \in s_{3}$, can be computed as

$$
\begin{aligned}
& \mathrm{P}\left[\theta_{k_{m}}^{j}=1 \mid \theta_{k+r_{j}}^{j}=1, \theta_{k^{\prime}}^{i}=0 \quad \forall k^{\prime} \in\left[k, k_{m}\right]\right]= \\
& \mathrm{P}\left[\nu_{k_{m}-1}^{i}>\nu_{k_{m}-1}^{j} \mid \theta_{k+r_{j}}^{j}=1, \theta_{k^{\prime}}^{i}=0 \quad \forall k^{\prime} \in\left[k, k_{m}\right]\right] \leq \\
& \frac{\mathrm{E}\left[\nu_{k_{m}-1}^{i} \mid \theta_{k^{\prime}}^{i}=0 \quad \forall k^{\prime} \in\left[k, k_{m}\right]\right]}{s_{k_{m}-1}^{j} \tau},
\end{aligned}
$$

where, the last expression follows from Markov's inequality, and $\nu_{k_{m}-1}^{j}=s_{k_{m}-1}^{j} \tau$, for arbitrary $s_{k_{m}-1}^{j} \in\{1, \ldots, h-1\}$. Exploiting the law of iterated expectation, we can compute (22), given the latest error vector $e_{k_{m}-1}^{i}$, and from (11):

$$
\frac{\mathrm{E}\left[\mathrm{E}\left[\nu_{k_{m}-1}^{i} \mid e_{k_{m}-1}^{i}\right] \mid \theta_{k^{\prime}}^{i}=0\right]}{s_{k_{m}-1}^{j} \tau}=\frac{\alpha_{k_{m}-1}^{i}}{s_{k_{m}-1}^{j} \tau\left\|e_{k_{m}-1}^{i}\right\|_{Q_{i}}^{2}}:=\mathrm{P}_{s_{3}} .
$$

Incorporating $\mathrm{P}_{s_{3}}$, we have for a sub-system $i \in s_{3}$

$$
\begin{aligned}
& \sum_{i \in s_{3}} \mathrm{P}_{s_{3}} \mathrm{E}\left[\left\|e_{k_{m}}^{i}\right\|_{Q_{i}}^{2} \mid e_{k_{m}-1}^{i}\right] \\
& =\sum_{i \in s_{3}} \mathrm{P}\left[\nu_{k_{m}-1}^{i}>\nu_{k_{m}-1}^{j} \mid \theta_{k^{\prime}}^{i}=0, \forall k^{\prime}\right] \mathrm{E}\left[\left\|e_{k_{m}}^{i}\right\|_{Q_{i}}^{2} \mid e_{k_{m}-1}^{i}\right] \\
& \leq \sum_{i \in s_{3}} \frac{\alpha_{k_{m}-1}^{i}\left\|\bar{A}_{i}^{c}\right\|_{2}^{2}}{s_{k_{m}-1}^{j} \tau} \\
& +\sum_{i \in s_{3}} \frac{\alpha_{k_{m}-1}^{i}\left[\left[\left\|\bar{A}_{i}^{c}\right\|_{2}^{2}+1\right] \operatorname{tr}\left(Q_{i} V_{i}\right)+\left\|C_{i}\right\|_{2}^{2} \operatorname{tr}\left(Q_{i} W_{i}\right)\right]}{s_{k_{m}-1}^{j} \tau \lambda_{i}},
\end{aligned}
$$

where, the last line follows as $\left\|e_{k_{m}-1}^{i}\right\|_{Q_{i}}^{2}>\lambda_{i}$. Extending the interval to $\left[k, k_{m}\right]$, we can upper-bound (16) as follows:

$$
\begin{aligned}
& \sum_{i=1}^{N+m} \mathrm{E}\left[\left\|e_{k_{m}}^{i}\right\|_{Q_{i}}^{2}\right] \leq \sum_{i \in s_{1}} \mathrm{E}\left[\left\|e_{k_{m}}^{i}\right\|_{Q_{i}}^{2}\right] \\
& \quad+\sum_{i \in s_{2}} \mathrm{E}\left[\left\|e_{k_{m}}^{i}\right\|_{Q_{i}}^{2}\right]+\sum_{i \in s_{3}} \mathrm{P}_{s_{3}} \mathrm{E}\left[\left\|e_{k_{m}}^{i}\right\|_{Q_{i}}^{2}\right] .
\end{aligned}
$$

As (14) holds over the interval $[k, k+N]$ within the scenarios $s_{1}$ and $s_{2}$, it also holds over longer intervals. Moreover, since (23) is uniformly bounded, (15) will be satisfied over $\left[k, k_{m}\right]$ by setting $\bar{\xi}^{\prime}$ greater than (23) and selecting $\bar{\xi}=\frac{\sum_{i \in s_{3}} \mathrm{P}_{s_{3}} \mathrm{E}^{[}\left[e_{k_{m}}^{i} \|_{Q_{i}}^{2} \mid e_{k}^{i}\right]}{\xi^{\prime}}<1$. Thus (15) is satisfied over the interval $\left[k, k_{m}\right]$ with $m$ collisions. It follows then from Lemma 1 that the described NCS possesses LSP.

\section{REFERENCES}

[1] J. P. Hespanha, P. Naghshtabrizi, and Y. Xu, "A survey of recent results in networked control systems," PROCEEDINGS-IEEE, vol. 95, no. 1, p. 138, 2007.

[2] G. C. Walsh and H. Ye, "Scheduling of networked control systems," Control Systems, IEEE, vol. 21, no. 1, pp. 57-65, 2001.

[3] K. Åström and B. Bernhardsson, "Comparison of Riemann and Lebesgue sampling for first order stochastic systems," in 41st IEEE Conf. on Decision and Control, pp. 2011-2016 vol.2, 2002.

[4] D. Dimarogonas, E. Frazzoli, and K. Johansson, "Distributed eventtriggered control for multi-agent systems," Automatic Control, IEEE Trans. on, vol. 57, pp. $1291-1297$, may 2012.

[5] X. Wang and M. Lemmon, "Event-triggering in distributed networked control systems," IEEE Trans. on Automatic Control, vol. 56, no. 3 , pp. 586-601, 2011.

[6] J. Lunze and D. Lehmann, "A state-feedback approach to event-based control," Automatica, vol. 46, no. 1, pp. 211-215, 2010.

[7] M. Rabi, G. Moustakides, and J. Baras, "Adpative sampling for linear state estimation," SIAM journal on control and optimization, 2009.

[8] A. Molin and S. Hirche, "Price-based adaptive scheduling in multiloop control systems with resource constraints," IEEE Trans. on Automatic Control, vol. 59, no. 12, pp. 3282-3295, 2014.

[9] M. Mamduhi, A. Molin, and S. Hirche, "Event-based scheduling of multi-loop stochastic systems over shared communication channels," in 21 st Int. Symp. on Mathematical Theory of Networks and Systems, pp. 266-273, 2014.

[10] A. Cervin and T. Henningsson, "Scheduling of event-triggered controllers on a shared network," in 47th IEEE Conf. on Decision and Control, pp. 3601-3606, 2008.

[11] R. Blind and F. Allgöwer, "Analysis of networked event-based control with a shared communication medium: Part II - slotted ALOHA," in Proc. 18th IFAC World Congress, 2011.

[12] A. Molin and S. Hirche, "On the optimality of certainty equivalence for event-triggered control systems," IEEE Trans. on Automatic Control, vol. 58, no. 2, pp. $470-474,2013$.

[13] G. C. Walsh, H. Ye, and L. G. Bushnell, "Stability analysis of networked control systems," IEEE Trans. on Control Systems Technology, vol. 10, no. 3, pp. 438-446, 2002.

[14] M. Mamduhi, D. Tolic, A. Molin, and S. Hirche, "Event-triggered scheduling for stochastic multi-loop networked control systems with packet dropouts," in 53rd IEEE Conf. on Decision and Control, pp. 2776-2782, 2014.

[15] M. Mamduhi, D. Tolic, and S. Hirche, "Decentralized event-based scheduling for shared-resource networked control systems," in 14th European Control Conf., pp. 941-947, 2015.

[16] M. Tabbara and D. Nesic, "Input-output stability of networked control systems with stochastic protocols and channels," IEEE Trans. on Automatic Control, vol. 53, no. 5, pp. 1160-1175, 2008.

[17] M. Donkers, W. Heemels, D. Bernardini, A. Bemporad, and V. Shneer, "Stability analysis of stochastic networked control systems," Automatica, vol. 48, no. 5, pp. 917-925, 2012.

[18] M. Vilgelm, M. Mamduhi, W. Kellerer, and S. Hirche, "Adaptive decentralized MAC for event-triggered networked control systems," in 19th Int. Conf. on Hybrid Systems: Computation and Control, 2016.

[19] F. Kozin, "A survey of stability of stochastic systems," Automatica, vol. 5, pp. 95-112, Jan. 1969. 